\title{
Leptin Receptor Signaling and the Regulation of Mammalian Physiology
}

\author{
Martin G. Myers, JR. \\ Section on Obesity, Research Division, Joslin Diabetes Center, and Harvard Medical School, \\ Boston, Massachusetts 02215
}

\begin{abstract}
While the hormone leptin and its receptor were discovered relatively recently, a great deal is already known about the molecular details of leptin receptor (LR) signaling and physiologic regulation. While multiple alternatively spliced LR isoforms exist, only the long ( $\mathrm{LRb}$ ) form associates with the Janus kinase 2 (Jak2) tyrosine kinase to mediate intracellular signaling. LRb initiates signaling via three major mechanisms: 1) $\mathrm{Tyr}_{985}$ of $\mathrm{LRb}$ recruits SH2-containing tyrosine phosphatase (SHP-2); 2) Tyr $_{1138}$ of LRb recruits signal transducer and activator of transcription 3 (STAT3); and 3) tyrosine phosphorylation sites on the receptor-associated Jak2 likely recruit numerous undefined signaling proteins. The $\mathrm{Tyr}_{985} \rightarrow$ SHP-2 pathway is a major regulator of extracellular signal-regulated kinase (ERK) activation during leptin signaling in cultured cells, while the $\mathrm{Tyr}_{1138} \rightarrow$ STAT3 pathway induces the feedback inhibitor, suppressor of cytokine signaling 3 (SOCS3), as well as important positive effectors of leptin action. The Jak2-dependent activation of the insulin receptor substrate (IRS) protein $\rightarrow$ phosphatidylinositol 3-kinase (PI3'-K) pathway appears to regulate membrane potential in LRb-expressing neurons and contributes to the regulation of feeding. The $\mathrm{Tyr}_{1138} \rightarrow$ STAT3 pathway mediates transcriptional regulation of the hypothalamic melanocortin pathway in vivo. This pathway is required for the regulation of appetite and energy expenditure by leptin. Interestingly, the $\mathrm{Tyr}_{1138} \rightarrow$ STAT3 pathway does not strongly regulate neuropeptide Y (NPY) and thus is not required for the control of reproduction and growth. Thus, other as-yet-undefined leptin receptor signals are central to these and perhaps other aspects of leptin action.
\end{abstract}

\section{Background}

\section{A. THE $o b$ and $d b$ LOCI}

Although leptin was identified only recently (1994), the history of leptin began several decades ago with the description of the obese $(o b)$ and diabetes $(d b)$ mouse models that spontaneously and independently arose in the mouse breeding program at the Jackson Laboratories (Flier, 1995; Friedman and Halaas, 1998). In the homozygous state $(o b / o b$ or $d b / d b)$, mutations at these loci cause hyperphagia and decreased metabolic rate (resulting in morbid obesity), predisposition to diabetes, and endocrine disturbances. While genetic studies mapped 
these two genes to separate loci, their relatedness was suggested early on by an intriguing set of parabiosis studies by Douglas Coleman in which the circulatory systems of pairs of wild-type, $o b / o b$, and $d b / d b$ mice were joined. Parabiosis of $o b / o b$ animals with either wild-type or $d b / d b$ mice abrogated the phenotype of $o b / o b$ mice, suggesting that the $o b$ gene product (missing in $o b / o b$ mice) was a circulating factor (a hormone) present in wild-type and $d b / d b$ mice. In contrast, neither wild-type nor $o b / o b$ mice could alter the phenotype of $d b / d b$ mice to which they were joined, suggesting that the $d b / d b$ mouse was insensitive to this factor. Coleman theorized that the receptor for the $o b$ factor was mutated in the $d b / d b$ mouse.

The predictions of these classic experiments were confirmed when the $o b$ gene was positionally cloned in a large mouse genetics study. The gene encodes a predicted secreted protein, termed leptin (Greek: leptos = thin) (Flier, 1995; Friedman and Halaas, 1998). Soon after the cloning of leptin, it was demonstrated that leptin is synthesized and secreted from adipocytes and that treatment of $o b / o b$ mice with leptin reversed their phenotype (Halaas et al., 1995). Treatment of $d b / d b$ mice with leptin was ineffective, confirming their insensitivity to this factor.

Following the cloning of leptin, the leptin receptor (LR) was cloned quickly by biochemical techniques employing labeled leptin (Tartaglia et al., 1995). Soon thereafter, the original $d b$ mutation from Jackson Laboratories and several other independently arising $d b$ mutations were shown to lie within the leptin receptor gene (lepr) (Chua et al., 1996).

\section{B. LEPTIN COMMUNICATES THE STATUS OF BODY ENERGY STORES TO THE CENTRAL NERVOUS SYSTEM}

Adipose cells are the primary source of leptin. Leptin production by fat cells in culture is stimulated by glucocorticoids and indicators of acute nutritional influx such as insulin and inhibited by the counter-regulatory hormones and their intracellular signaling mediators (MacDougald et al., 1995; Rentsch and Chiesi, 1996; Slieker et al., 1996). This regulation of leptin production by insulin (feeding $\rightarrow$ increased insulin $\rightarrow$ increased leptin) and counter-regulatory hormones (fasting $\rightarrow$ increased counter-regulatory hormones $\rightarrow$ decreased leptin) suggests that leptin might serve as an indicator of energy balance (i.e., that increased energy stores yield increased leptin levels). Indeed, circulating leptin levels strongly correlate with body adiposity and changes in acute nutritional status (Frederich et al., 1995; Maffei et al., 1995; Considine et al., 1996).

The stimulation of leptin production by glucocorticoids appears counterintuitive in this light, however, since circulating glucocorticoids are important mediators of the stress and starvation responses. Indeed, while plentiful data suggest that insulin is a critical mediator of increased leptin production in vivo, 
the diurnal pattern of leptin levels and numerous other data argue against an important role for circulating glucocorticoids in the stimulation of leptin production (Licinio et al., 1997). In contrast, adipocyte-produced autocrine- or paracrine-acting glucocorticoids may be involved, since the production of glucocorticoids by adipocytes increases with the accumulation of triglycerides. Mouse models with increased glucocorticoid production specifically in adipocytes have elevated leptin levels (Bornstein et al., 1997).

Circulating leptin levels thus reflect the status of body energy reserves. Leptin regulates energy balance throughout the body by controlling processes involved in energy intake and utilization. Leptin regulates energy expenditure via the reproductive, growth, autonomic, and other axes of the neuroendocrine system as well as by the immune system (Ahima et al., 1996; Lord et al., 1998) (Figure 1). Thus, in times of inadequate energy stores (e.g., starvation), leptin

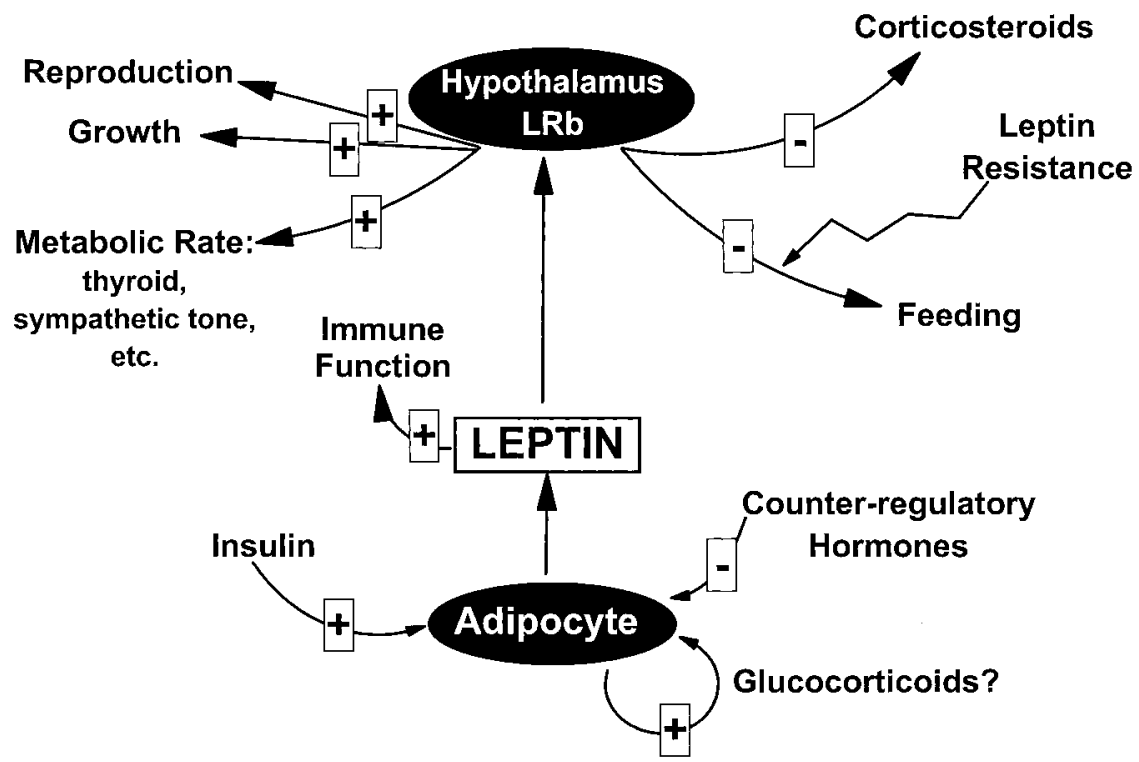

FIG. 1. Leptin signals the status of body energy stores to the hypothalamus and neuroendocrine system. Leptin production in adipocytes is regulated by indicators of acute nutritional status, such as insulin and counter-regulatory hormones, as well as by fat content (perhaps signaled via the autocrine/paracrine production of glucocorticoids). Leptin heightens immune responsiveness by direct effects upon the immune system and regulates feeding and neuroendocrine function by activating the long $(\mathrm{LRb})$ form of its receptor in the brain, most notably in the hypothalamus. Adequacy of leptin levels (reflecting adequate nutrition) suppresses feeding and the hypothalamic drive to produce glucocorticoids as well as permits the expenditure of energy by the reproductive, growth, and thyroid axes. Leptin resistance in common obesity appears to most strongly impact the ability of leptin to inhibit feeding. 
levels fall; these low leptin levels enhance appetite and decrease energy utilization. Conversely, with adequate energy stores, high leptin levels decrease the drive to eat and permit utilization of energy by the above-mentioned systems.

Lack of the leptin signal in mice (and humans) genetically null for leptin ( ob/ob mice) or the leptin receptor (LR) ( $d b / d b$ mice) results in obesity secondary to increased feeding and decreased energy utilization (Montague et al., 1997; Clement et al., 1998; Elmquist et al., 1998; Friedman and Halaas, 1998). These animals also display a phenotype reminiscent of the neuroendocrine starvation response, including hypothyroidism, decreased growth, and infertility, in addition to decreased immune function. Indeed, exogenous leptin replacement during food restriction restores each of these functions as well as decreases appetite (Ahima et al., 1996).

\section{LEPTIN RECEPTORS AND SITES OF LEPTIN ACTION}

Multiple LR isoforms exist; all are products of a single lepr gene and result from alternative mRNA splicing and/or proteolytic processing (Chua et al., 1997; Tartaglia, 1997). LR isoforms fall into three classes: secreted, short, and long. The secreted forms are alternative splice products (e.g., the murine LRe) or proteolytic cleavage products of membrane-bound LR forms: These contain only extracellular leptin-binding domains and complex with circulating leptin, perhaps regulating free leptin concentrations (Ge et al., 2002). Short- (e.g., LRa) and long-form $(\mathrm{LRb})$ receptors contain identical extracellular and transmembrane domains as well as the same first 29 intracellular amino acids and diverge in sequence secondary to alternative splicing of $3^{\prime}$ exons. LRb is highly conserved among species and possesses an intracellular domain of approximately 300 residues (Chua et al., 1997; Tartaglia, 1997).

While the function of the short LR forms remains unclear, LRb is critical for leptin action. Indeed, the originally described $d b / d b$ mice lack only LRb (as a consequence of a mutation that results in mis-splicing of the LRb message) but exhibit a phenotype indistinguishable from that of leptin-deficient $o b / o b$ animals and of $d b^{3 J} / d b^{3 J}$ mice (which are deficient in all LR isoforms) (Chua et al., 1997; Tartaglia, 1997; Friedman and Halaas, 1998).

Much of leptin's action is attributable to effects in the central nervous system (CNS), especially in the basomedial hypothalamus, the site of highest LRb expression (Elmquist et al., 1998,1999). Here, leptin acts on neurons that regulate levels of circulating hormones (e.g., thyroid hormone, sex steroids, growth hormone) (Elmquist et al., 1999; Inui, 1999). Leptin action on these hypothalamic neurons also regulates the activity of the autonomic nervous system, although direct leptin action on brainstem LRb-expressing neurons may play a role in this and other leptin actions (Elmquist et al., 1997; Grill et al., 2002). Leptin action on the immune system appears to result from direct action on 
LRb-expressing T cells (Lord et al., 1998). Leptin also may regulate glucose homeostasis (independently of effects on adiposity). Leptin regulates glycemia at least partly via the CNS but also may directly regulate some metabolic tissues (Liu et al., 1988; Kieffer et al., 1997; Kulkarni et al., 1997; Burcelin et al., 1999).

\section{LEPTIN RESISTANCE AND OBESITY}

Over one quarter of adult Americans are obese. The incidence of obesity continues to rise in the United States as well as in other industrialized nations. Obesity is a major risk factor for type 2 diabetes, cardiovascular disease, and some forms of cancer (Roth, 1998). Since administration of leptin to rodents decreases food intake and increases energy expenditure, resulting in loss of fat mass, leptin initially was hailed as a potential cure for obesity (Tartaglia, 1997; Elmquist et al., 1998,1999; Friedman and Halaas, 1998; Inui, 1999).

With the exception of rare human patients with genetic leptin deficiency, however, circulating leptin levels correlate well with body mass index (BMI) and total body fat mass. Hence, obese individuals have elevated circulating leptin levels but this abundant leptin fails to mediate weight loss, suggesting that most human obesity represents a form of leptin resistance. Indeed, although therapy with exogenous leptin does augment weight loss, the effects of leptin are modest at the doses that have been tested. A number of potential mechanisms have been postulated to underlie leptin resistance, including defects in leptin access into the brain, in LRb signaling, or in pathways/neurons that mediate downstream leptin action. We predict that defects in LRb signaling or downstream effectors will underlie leptin resistance, since leptin may gain direct access (without need for transport across the blood-brain barrier) to neurons in the basomedial hypothalamus that are important for the regulation of energy balance.

\section{LR Signaling Systems}

\section{A. LEPTIN SIGNALING AT THE OUTSET}

My graduate training in the laboratory of Morris White, Ph.D. at the Joslin Diabetes Center focused on the role(s) played by insulin receptor substrate-1 (IRS-1) in cellular insulin signaling. After defending my thesis, I returned to medical school to complete the clinical component of my training. I realized that I preferred to focus on research in the long term, as opposed to continuing with clinical practice. Thus, it was in the late winter and early spring of 1997 that I found myself finishing my medical education and thinking about a new scientific path to follow to establish my independence. Possessing a background in basic tyrosine kinase signaling, I wished to play to this strength as well as to continue working in a direction relevant to diabetes and the control of metabolism. It was 
clear that switching away from insulin signaling and IRS-1 would facilitate my transition to independence. Although I considered for a time studying signaling by cytokines in immune function, the initial cloning of the leptin receptor just had been reported to great fanfare (Tartaglia et al., 1995). LRb was clearly a type I cytokine receptor that mediated cell signaling via a member of the Jak family of tyrosine kinases. The obese and diabetic phenotype of mice deficient in leptin or $\mathrm{LRb}$ made it clear that it was a metabolically relevant target. While the hubbub surrounding leptin action was huge (and remains high), it was clear that most investigators had priorities other than studying the intricate details of LR signaling and cell biology, although this area would likely prove to be interesting and important.

The homology of LRb with other type I cytokine receptors (e.g., the gp 130 subunit of the interleukin (IL)-6 receptor family) suggests a number of signaling functions that were tested quickly by a number of groups: leptin binding to LRb activates Jak kinases and mediates cytokine receptor-like signals, including those mediated by the latent transcription factor, signal transducer and activator of transcription 3 (STAT3) (Baumann et al., 1996; Tartaglia, 1997; White et al., 1997). A specific tyrosine residue $\left(\mathrm{Tyr}_{1138}\right)$ in the intracellular domain of $\mathrm{LRb}$ mediates the activation of STAT3.

We felt that LRb was likely to mediate other important signals as well and set out to identify them. In general, tyrosine kinase-based signaling is mediated by the recruitment of signaling molecules to tyrosine-phosphorylated motifs on the receptor and/or tyrosine kinase (Koch et al., 1991) — in this case, on the intracellular domain of LRb and its associated Jak kinase(s). This recruitment of downstream signaling proteins generally is mediated by specialized phosphotyrosine binding domains (e.g., src homology 2 (SH2) domains) in the signaling proteins. These $\mathrm{SH} 2$ domains generally require the presence of phosphotyrosine in the target protein for high-affinity binding but also recognize surrounding amino acid residues. Thus, each tyrosine phosphorylation site on a receptor recruits specific SH2 domain-containing proteins, based upon the amino acid motif in which the phosphotyrosine residue is located. In this manner, the residues Met-Pro-Gln (MPQ) following $\mathrm{Tyr}_{1138}$ of LRb dictate binding of STAT3 when Tyr $_{1138}$ becomes phosphorylated (Baumann et al., 1996; Tartaglia, 1997; White et al., 1997).

Understanding the signals mediated by LRb requires identifying the tyrosine residues within the LRb/Jak kinase complex that are phosphorylated during signaling and subsequently determining the signaling proteins that interact with these sites. While the identification of LRb phosphorylation sites should be relatively straightforward, identifying phosphorylation sites on the associated Jak kinase is more complicated, given that it requires identifying the Jak kinase isoform(s) utilized by LRb (Ihle, 1995) as well as sorting through a greater number of tyrosine phosphorylation sites (Feng et al., 1997). Furthermore, in 
order to understand the failure of short LR isoforms to compensate for the lack of LRb in $d b / d b$ animals, it is critical to define their ability to mediate Jakdependent signaling.

In order to avoid potential artifacts introduced by the endogenous (mostly short-form) LR isoforms expressed in most cells, we employ a chimeric receptor (ELR) that contains the extracellular ligand-binding domain of the erythropoeitin (Epo) receptor fused to the transmembrane and intracellular domains of LR forms (Banks et al., 2000). The EpoR is expressed on few cells or cell lines, so ELR permits us to use Epo to stimulate signaling via various LR intracellular domains without triggering endogenous receptors.

\section{B. JAK KINASE SELECTIVITY AND UTILIZATION BY LR ISOFORMS}

There are four known members of the Jak family: Jak1-3 and Tyk2. Jak1, Jak2, and Tyk2 are widely expressed, whereas Jak3 is found only in cells of the hematopoeitic/immune systems (Ihle, 1995). We expressed ELR in 32D myeloid progenitor cells and assessed its ability to mediate the activation of the commonly expressed Jak kinases, using the endogenous (Jak-promiscuous) IL-3 receptor as a positive control for the activation of each kinase. This analysis demonstrated that the intracellular domain of ELR activated only Jak2, not Jak1 or Tyk2 (Kloek et al., 2002). Furthermore, while disruption of Jak2 expression in fibrosarcoma cells abrogated ELR signaling, disruption of Jak1 or Tyk2 had no effect. Thus, Jak2 represents the unique Jak kinase involved in signaling by the intracellular domain of LRb.

All functional cytokine receptors contain a proline-rich "Box 1" motif that is required for Jak kinase interaction and activation. Additional less-conserved sequences (sometimes referred to as "Box 2") COOH-terminal to Box 1 are also important for Jak kinase interactions and likely function in Jak kinase isoform selectivity (Ihle and Kerr, 1995; Taga and Kishimoto, 1997). Our deletion analysis demonstrated that, in addition to Box 1 sequences, intracellular residues 31-36 of LRb (i.e., immediately downstream of the alternative splice junction following amino acid 29) are required for Jak2 activation (Kloek et al., 2002). Interestingly, we observed that high-level overexpression of Jak kinases by transient transfection could decrease the stringency of the requirement for residues 31-36, consistent with the ability of others to observe (relatively weak) LRb-Jak1 and LRa-Jak2 signaling under transient transfection conditions (Bjorbaek et al., 1997). These results suggests a model in which Box 1 is absolutely required for all cytokine receptor-Jak kinase interactions, with the more-flexible role (at least under experimental conditions) for residues homologous to intracellular amino acids 31-36 of LRb in determining the specificity of the Jak kinase that interacts with a particular receptor. Indeed, homology between the Box 2 regions of $\mathrm{LRb}$ and other Jak2-associated cytokine receptors suggests that 
a loosely conserved $\mathrm{E} / \mathrm{N}-\mathrm{X}_{0-2} \mathrm{E} / \mathrm{N}-\mathrm{X}_{0-2} \mathrm{~L} / \mathrm{I}$ motif mediates Jak2 association (Kloek et al., 2002).

This motif is absent from all described short LR isoforms, explaining the inability of these molecules to mediate leptin action in $d b / d b$ animals (Tartaglia, 1997; White et al., 1997; Kloek et al., 2002). The lack of Jak2 signaling by short LR isoforms suggests we cannot rule out, on the basis of the failure of these receptors to transmit any leptin signal, the possibility that Jak2 may be capable of mediating some physiologic leptin actions in the absence of LRb tyrosine phosphorylation sites.

\section{LRb TYROSINE PHOSPHORYLATION}

There are three conserved tyrosine residues on the intracellular domain of LRb: $\mathrm{Tyr}_{985}, \mathrm{Tyr}_{1077}$, and $\mathrm{Tyr}_{1138}$ (Tartaglia, 1997; White et al., 1997; Banks et al., 2000). In the human long-form LR, two additional intracellular tyrosine residues exist, albeit within motifs that do not appear to be particularly hydrophilic and accessible to kinases (Tartaglia et al., 1995). In order to determine whether any of these sites might be candidates for phosphorylation, we generated a bacterial fusion protein containing all of the tyrosine residues of the intracellular domain of the human long-form LR (except the $\mathrm{Tyr}_{1138}$ homologue) and subjected it to phosphorylation with recombinant Jak2. Analysis of the resulting phosphorylated glutathione-S-transferase (GST)-LR demonstrated tyrosine phosphorylation of the $\mathrm{Tyr}_{985}$ homologue but not of any other sites, suggesting that $\mathrm{Tyr}_{985}$ and $\mathrm{Tyr}_{1138}$ might be the unique sites of LRb tyrosine phosphorylation (M.G. Myers, Jr., unpublished observations).

In order to examine the phosphorylation of tyrosine residues on the intracellular tail of LRb in intact cells, we mutated the three LRb tyrosines individually and in combination and examined the phosphorylation of the mutant receptors during signaling in 293 cells (Banks et al., 2000). This analysis confirmed that $\mathrm{Tyr}_{985}$ and $\mathrm{Tyr}_{1138}$ are phosphorylated during receptor signaling but $\mathrm{Tyr}_{1077}$ is not phosphorylated and does not contribute to signaling. Thus, three primary intracellular signaling pathways emanate from LRb (Figure 2): those originating directly from Jak2 tyrosine phosphorylation sites, from $\mathrm{Tyr}_{985}$ of $\mathrm{LRb}$, and from $\mathrm{Tyr}_{1138}$ of LRb.

\section{SIGNALING VIA TYR ${ }_{985}$ MEDIATES SHP-2 RECRUITMENT AND ERK ACTIVATION}

We and others had noted the activation of the ERK kinases during signaling by the intracellular domain of LRb (Bjorbaek et al., 1997; Banks et al., 2000). In order to understand the mechanism for this activation, we employed our ELR isoforms containing tyrosine substitution mutations. We noted that mutation of

$\mathrm{Tyr}_{985}$ inhibited ERK activation by approximately $70 \%$ and that the remaining 


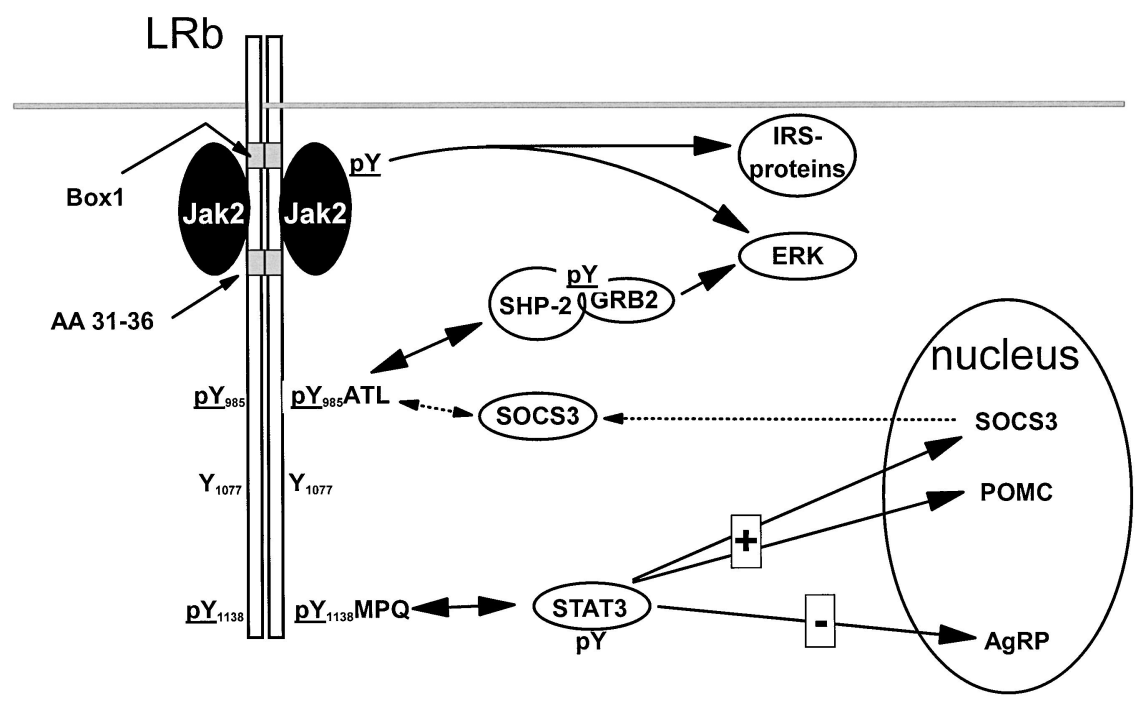

FIG. 2. Intracellular signaling by LRb. Leptin binding to the extracellular domain of the LRb dimer activates the Jak2 tyrosine kinase that associates with $\mathrm{LRb}$ via the Box 1 motif and intracellular amino acids 31-36 of LRb. Activated Jak2 tyrosine phosphorylates itself and $\mathrm{Tyr}_{985}$ and $\mathrm{Tyr}_{1138}$ on the intracellular tail of LRb. Phosphorylated $\mathrm{Tyr}_{1138}$ binds and mediates the phosphorylationdependent activation of signal transducer and activator of transcription 3 (STAT3), which activates transcription of suppressor of cytokine signaling 3 (SOCS3) (a feedback inhibitor of LRb signaling) and pro-opiomelanocortin (POMC) (an anorexigenic neuropeptide) and inhibits the transcription of agouti-related protein (AgRP) (an orexigenic neuropeptide). Phosphorylated $\mathrm{Tyr}_{985}$ recruits the SH2-containing tyrosine phosphatase SHP-2, which is itself phosphorylated and binds growth factor receptor binding 2 (Grb-2) to activate the signaling pathway that culminates in extracellular signal-regulated kinase (ERK) activation. During prolonged stimulation, after induction of SOCS3 protein, phosphorylated $\operatorname{Tyr}_{985}$ also mediates binding of SOCS3. Signals mediated via unidentified tyrosine phosphorylation sites on LRb include the tyrosine phosphorylation of insulin receptor substrate (IRS) proteins and a minor component of ERK activation.

$30 \%$ of ERK activation required Jak2 interaction but none of the tyrosine phosphorylation sites on LRb. This suggested that a minor pathway to ERK activation is mediated independently of LRb phosphorylation, presumably via tyrosine phosphorylation sites on Jak2 (Banks et al., 2000).

The canonical mechanism by which tyrosine kinases activate ERK is via the SH2 domain-containing adapter protein, growth factor receptor binding (Grb)-2. Grb-2 recruitment by tyrosine kinase signaling complexes mediates the activation of the small G protein, p21ras, to stimulate the upstream activators of ERK (Lowenstein et al., 1992; Myers et al., 1994; Banks et al., 2000). In order to understand the mechanism of ERK activation by $\mathrm{Tyr}_{985}$, we examined tyrosyl phosphoproteins associated with the adapter protein, Grb-2, during ELR signal- 
ing and discovered an $\sim 75-\mathrm{kDa}$ Grb-2-associated tyrosyl phosphoprotein (Banks et al., 2000). Taking a candidate approach, we showed that this $75-\mathrm{kDa}$ protein is identical to the SH2 domain-containing tyrosine phosphatase SHP-2 and that $\mathrm{Tyr}_{985}$ directly recruits SHP-2 via its $\mathrm{COOH}$-terminal SH2 domain (Banks et al., 2000; Bjorbaek et al., 2001; Kloek et al., 2002). Indeed, the YATL motif in which $\mathrm{Tyr}_{985}$ lies is predicted to bind to the $\mathrm{COOH}$-terminal $\mathrm{SH} 2$ domain of SHP-2 (Songyang et al., 1993; De Souza et al., 2002). Thus, phosphorylation of $\mathrm{LRb} \mathrm{Tyr}_{985}$ mediates the recruitment of SHP-2, which is then phosphorylated and, in turn, recruits Grb-2 to mediate activation of the p21ras $\rightarrow$ ERK cascade.

\section{E. SIGNALING VIA TYR TY $_{138} \rightarrow$ STAT3 MEDIATES TRANSCRIPTION OF SOCS3 AND FEEDBACK INHIBITION ON LRb SIGNALING}

The role of $\mathrm{Tyr}_{1138}$ phosphorylation in recruiting STAT3 to the LRb/Jak2 complex was suggested by homology between LRb and other cytokine receptors of the IL-6 receptor family. Indeed, mutation of this site abrogates STAT3 signaling by LRb (Baumann et al., 1996; Tartaglia, 1997; White et al., 1997). We thus utilized receptors mutant for $\mathrm{Tyr}_{1138}$ to investigate potential roles for STAT3 in the regulation of gene expression, demonstrating that this pathway induces expression of SOCS3, which is induced by prolonged LRb activation and mediates feedback inhibition of LRb signaling (Bjorbaek et al., 1998,2000; Banks et al., 2000). We furthermore examined the mechanism by which SOCS3 mediates feedback inhibition on signaling by the intracellular domain of LRb. We demonstrated that SOCS3 interacts via its $\mathrm{SH} 2$ domain with phosphorylated Tyr $_{985}$ of LRb and that sensitive blockade of LRb $\rightarrow$ STAT3 signaling is mediated by this interaction (Bjorbaek et al., 2000). Our recent unpublished results suggest that another LRb $\rightarrow$ STAT3-dependent mechanism of feedback inhibition of LRb signaling may directly block Jak2 activation.

\section{F. SIGNALS MEDIATED BY JAK2 AND FUTURE DIRECTIONS IN LRb SIGNALING}

Jak2 tyrosine phosphorylation during LRb stimulation mediates some signals independently of tyrosine phosphorylation sites on LRb (e.g., a portion of ERK activation, IRS protein phosphorylation) (Banks et al., 2000). Unfortunately, most Jak2 tyrosine phosphorylation sites have not been defined, impairing our understanding of the mechanisms by which Jak2-dependent signals are mediated. Identifying tyrosine phosphorylation sites on Jak2 and defining their roles in intracellular signaling will be critical to understanding the breadth and depth of LRb signaling. 


\section{Leptin Signals in the Regulation of Mammalian Physiology}

\section{A. LEPTIN ACTION IN THE HYPOTHALAMUS}

While LRb is expressed at other sites, the highest levels of LRb expression in the body are found in neurons of the nuclei of the basomedial hypothalamus — including the arcuate (ARC), dorsomedial hypothalamic (DMH), and ventromedial hypothalamic (VMH) nuclei (Elmquist et al., 1998). Chemical or physical ablation of these nuclei results in increased feeding and neuroendocrine abnormalities that are similar to the phenotypes of $d b / d b$ or $o b / o b$ mice, suggesting that these hypothalamic nuclei (which make up the so-called "satiety center") are critical sites of leptin action (Elmquist et al., 1999; Schwartz et al., 2000).

Within the nuclei of the basomedial hypothalamus, LRb is expressed at its highest levels in the ARC. Within the ARC, LRb is found in at least two distinct populations of neurons (Figure 3): 1) neurons that coexpress neuropeptide $\mathrm{Y}$ (NPY) and agouti-related peptide (AgRP) and 2) neurons that express pro-

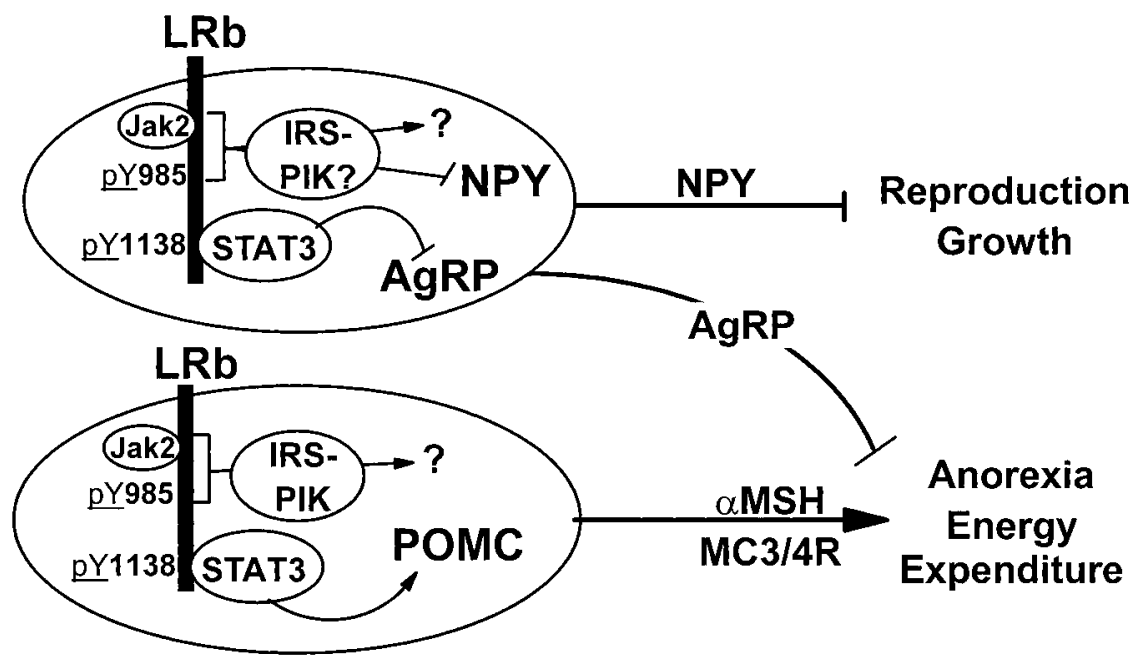

FIG. 3. Neuroendocrine regulation in the arcuate nucleus (ARC) by LRb signals. In the $\mathrm{LRb} / \mathrm{POMC}$ neuron in the ARC, the $\mathrm{Tyr}_{1138} \rightarrow$ STAT3 signal mediates the induction of POMC, which is processed to alpha melanocyte-stimulating hormone $(\alpha \mathrm{MSH})$ to activate anorexia and energy expenditure via the melanocortin receptors, MC3R and MC4R. The function of other $\mathrm{LRb} / \mathrm{Jak} 2$ signals, such as IRS proteins and phosphatidylinositol 3-kinase (IRS-PI3-K), remain undefined in this neuron. In the ARC LRb/neuropeptide Y (NPY)/AgRP-expressing neuron, STAT3 signaling contributes to the repression of AgRP and the de-repression of melanocortin action, while other signals (possibly including IRS-PI3-K) function to repress NPY, disinhibiting the growth and reproductive axes. Other IRS-PI3-K-mediated signals may include the regulation of membrane potential. 
opiomelanocortin (POMC) (Elmquist et al., 1999; Schwartz et al., 2000). POMC is processed to alpha melanocyte-stimulating hormone $(\alpha \mathrm{MSH})$ in the $\mathrm{LRb} /$ POMC neuron. $\alpha \mathrm{MSH}$ mediates a powerful anorectic (appetite-suppressing) signal; LRb stimulates the expression of POMC and activates the LRb/POMC neuron (Schwartz et al., 2000; Cowley et al., 2001). AgRP is an antagonist of $\alpha \mathrm{MSH}$ signaling and NPY is itself an orexigenic (appetite-stimulating) hormone that also acts to suppress the central LRb growth and reproductive axes (Erickson et al., 1996b; Fan et al., 1997; Seeley et al., 1997; Bates et al., 2003). Leptin acts via LRb to inhibit the NPY/AgRP neurons and to suppress expression of these neuropeptides. Thus, LRb signaling stimulates the production of anorectic neuropeptides and suppresses levels of orexigenic peptides. Conversely, when leptin action is decreased or deficient (e.g., starvation, $o b / o b$ or $d b / d b$ mice), appetite is stimulated via the suppression of anorectic neuropeptides (e.g., POMC) and by increased expression of orexigenic peptides (e.g., NPY, AgRP) (Elmquist et al., 1999; Schwartz et al., 2000). LRb-expressing ARC NPY/AgRP and/or POMC neurons also regulate energy expenditure and other elements of neuroendocrine function (Ahima et al., 1996). Other distinct populations of LRb-expressing neurons may be found in the ARC (Jureus et al., 2000). The neurochemical properties of LRb-expressing neurons in the DMR, VHM, and elsewhere (including the brainstem) are poorly defined.

\section{B. IRS PROTEIN/PI3'-K SIGNALING IN THE CONTROL OF PHYSIOLOGY BY LEPTIN}

Thus far, we have been involved in implicating two LRb signaling pathways in leptin action: STAT3 (see below) and the IRS protein-phosphatidylinositol 3-kinase (PI3'-K) pathway (Niswender et al., 2001; Bates et al., 2003).

First described as insulin receptor substrates, the IRS proteins (IRS 1-4) are members of a class of intracellular signaling molecules termed docking proteins that are phosphorylated by a number of activated tyrosine kinases (e.g., insulin receptor, some cytokine receptors) (Myers and White, 2002). Docking proteins, including the IRS proteins, are devoid of enzymatic activity but are phosphorylated on multiple tyrosine residues to mediate $\mathrm{SH} 2$-protein recruitment and downstream signaling. While IRS proteins contain tyrosine phosphorylation sites in numerous motifs that recruit several different $\mathrm{SH}$ 2-proteins, the majority of sites lie in YMXM motifs that bind and activate PI3'-K.

The first, albeit indirect, evidence for a potential role of the IRS protein $\rightarrow$ PI3'-K pathway in leptin action came from the phenotype of the IRS-2 null (IRS-2 ${ }^{-l-}$ ) mouse (Withers et al., 1998). In addition to other defects, IRS-2 ${ }^{-/-}$ animals display increased feeding and decreased metabolic rate in the presence of increased adiposity and circulating leptin, suggesting functional leptin resis- 
tance (although not as severe as in $d b / d b$ animals). No such phenotype has been noted in animals null for any of the other three IRS proteins (Araki et al., 1994).

Blockade of PI3'-K activity abrogates leptin-mediated hyperpolarization and inhibition of (presumably) LRb/NPY/AgRP hypothalamic neurons (Spanswick et al., 1997b; Harvey et al., 2000). Furthermore, leptin stimulates IRS-2-associated PI3'-K activity in the hypothalamus and pharmacological blockade of PI3'-K activity in the hypothalamus blocks the anorectic effect of leptin in vivo (Niswender et al., 2001).

Importantly, inhibition of PI3'-K does not alter the anorectic effect of melanocortin agonists that operate downstream of LRb neurons, suggesting that this effect of $\mathrm{PI} 3^{\prime}-\mathrm{K}$ blockade is specific for LRb neurons. PI3'-K activity also is required for leptin-regulated sympathetic nervous system function (Rahmouni et al., 2003).

As in vivo (Niswender et al., 2001), we observe that LRb stimulation mediates the tyrosine phosphorylation of IRS proteins and activation of the PI3'-K pathway in cultured cells. This signal is mediated by Jak2 independently of tyrosine phosphorylation sites on LRb (M.G. Myers, Jr., et al., unpublished observations).

The recruitment of IRS proteins/PI3'-K by Jak2 is less robust than by the insulin receptor, however. Indeed, animals with decreased expression of neuronal insulin receptors (NIRKO mice) display a modest obesity phenotype, similar to the phenotype of IRS-2 $2^{-1-}$ mice (Bruning et al., 2000). Also, insulin activates the IRS-2 $\rightarrow$ PI3'-K pathway in the hypothalamus and PI3'-K activity is required for the anorectic activity of insulin in the brain (Niswender et al., 2003). Thus, both leptin and insulin stimulate hypothalamic IRS-2 $\rightarrow$ PI3'-K signaling and both require $\mathrm{PI} 3^{\prime}-\mathrm{K}$ activity for their anorectic functions. Although the relative contribution of insulin- vs. leptin-stimulated PI3'-K to functional (anorectic) hypothalamic signaling remains difficult to dissect, the importance of the IRS protein $\rightarrow \mathrm{PI}^{\prime}{ }^{\prime}-\mathrm{K}$ pathway is clear, as will be the understanding of the potential role of this pathway in functional leptin resistance. It will be interesting to determine the neuropeptide phenotype of the neuronal population(s) in which $\mathrm{PI} 3^{\prime}-\mathrm{K}$ activity is required for the anorectic actions of leptin and insulin as well as the role of PI $3^{\prime}-\mathrm{K}$ in other leptin functions (e.g., neuroendocrine and immune regulation).

\section{LRb $\rightarrow$ STAT3 SIGNALING IN THE CONTROL OF PHYSIOLOGY BY LEPTIN}

We have directly addressed the contribution of the LRb $\rightarrow$ STAT3 pathway to physiology by studying homologously targeted "knock-in" mice in which LRb is replaced by a mutant molecule $\left(\mathrm{LRb}^{\mathrm{S} 1138}\right)$ that contains a substitution mutation of $\operatorname{Tyr}^{1138}$ (the STAT3 binding site) (Bates et al., 2003). While LRb ${ }^{\text {S1138 }}$ fails to 
mediate activation of STAT3 during leptin signaling, this mutant regulates all other LRb signaling pathways normally. This approach ensures that the expression pattern and levels of $\mathrm{LRb}^{\mathrm{S} 1138}$ mirror that of wild-type $\mathrm{LRb}$, so alterations in physiology in the resulting animals are secondary to lack of LRb $\rightarrow$ STAT3 signaling rather than due to a failure of correct LRb expression. Like $d b / d b$ animals, mice homozygous for $\mathrm{LRb}^{\mathrm{S} 1138}(s / s)$ display hyperphagia and decreased energy expenditure, resulting in massive, early-onset obesity associated with increased serum leptin levels. Thus, the LRb $\rightarrow$ STAT3 signal is central to the regulation of body weight by leptin and dysfunction of this signal generates leptin resistance.

Important differences exist between the phenotypes of $s / s$ mice and $d b / d b$ mice, however (Bates et al., 2003). While $d b / d b$ animals are infertile and demonstrate decreased linear growth, $s / s$ mice are fertile and demonstrate increased linear growth, compared to wild-type animals.

In order to understand the neurochemical basis for the $s / s$ phenotype, we analyzed hypothalamic neuropeptide expression, revealing that, like $d b / d b$ mice, $s / s$ mice have decreased POMC and increased AgRP mRNA levels in the hypothalamus. In contrast, while $d b / d b$ animals display dramatic induction of hypothalamic NPY mRNA, levels of NPY message are near normal in $s / s$ animals. These data suggest that LRb $\rightarrow$ STAT3 signaling is a critical regulator of hypothalamic melanocortin action and that dysregulated melanocortin signaling (as opposed to alterations in NPY) accounts for the obesity of $s / s$ animals. Additionally, non-STAT3 LRb signals are critical regulators of NPY expression in the LRb/NPY neuron.

These results are consistent with the proposed role for NPY in suppressing the hypothalamic growth and gonadal axes; thus, the increased NPY signaling in $o b / o b$ and $d b / d b$ mice may only modestly increase feeding but may be primarily responsible for infertility and growth retardation in these mouse models. Indeed, the phenotype of $o b / o b$ mice null for NPY $\left(o b / o b, \mathrm{Npy}^{-/-}\right)$displays important similarities with the $s / s$ phenotype (Erickson et al., 1996a): both show improved function of the hypothalamic/gonadal axis and increased linear growth with only modestly attenuated obesity, compared to $o b / o b$ and $d b / d b$ mice. The increased linear growth observed in these models likely results from the absolute lack of NPY in ob/ob, $\mathrm{Npy}^{-/-}$animals and the hyperactivation of non-STAT3 LRb signals (e.g., signals that inhibit the release of NPY) in hyperleptinemic $s / s$ animals, as is observed in most forms of human obesity. Thus, functional leptin resistance in humans could stem from inhibition of pathways controlled by LRb $\rightarrow$ STAT3 action.

Returning to the concept of leptin resistance in common forms of obesity, one would expect that dysregulation of the NPY pathway would result in neuroendocrine abnormalities (e.g., infertility, growth retardation) that are not generally observed in obesity. In contrast, alterations in STAT3-mediated path- 
ways (e.g., melanocortin action) could generate a phenotype of impaired energy balance coupled with relatively normal neuroendocrine function.

\section{FUTURE DIRECTIONS IN LRb SIGNALING AND PHYSIOLOGY}

Our data from $s / s$ animals demonstrate that LRb-STAT3 signaling is critical for the transcriptional regulation of melanocortin action in the ARC but not for the regulation of NPY. Additionally, STAT3 is not likely to be involved in the regulation of membrane potential in ARC neurons, as leptin-regulated membrane potential is too rapid to be transcriptionally mediated (Spanswick et al., 1997b; Cowley et al., 2001).

Note that the phenotype of the $s / s$ animals does not suggest the physiologic irrelevance of non-STAT3 pathways to the control of feeding and energy expenditure, only that STAT3 signaling is important for the regulation of energy homeostasis. We suggest that the Jak2 $\rightarrow$ IRS protein $\rightarrow$ PI3'-K pathway represents a major STAT3-independent mediator of LRb action. Data from numerous laboratories suggest that $\mathrm{PI} 3^{\prime}-\mathrm{K}$ action regulates membrane potential in the LRb/NPY neuron (Spanswick et al., 1997a; Harvey et al., 2000). PI3'-K action similarly may control membrane potential in the LRb/POMC neuron. Although this possibility remains untested, one reasonable hypothesis is that PI3'-K action may control the repression of NPY by LRb. However, it is also possible that $\mathrm{Tyr}_{985} / \mathrm{SHP}-2$-mediated signals or other uncharacterized signals mediated via Jak2 tyrosine phosphorylation sites may control NPY expression and/or membrane potential. The analysis of Jak2 and $\mathrm{Tyr}_{985}$-mediated signals in $\mathrm{LRb}$ action in vitro and in vivo will represent an important next step in understanding the mechanisms by which LRb regulates mammalian physiology.

\section{ACKNOWLEDGMENTS}

Too many people have contributed to the work described here to adequately acknowledge them all; some require special recognition, however. Thanks to Sarah Bates, Ph.D., the talented and energetic scientist who drives our investigation of leptin signaling in the regulation of mouse physiology. Thanks to Alex Banks, Christian Bjorbaek, Ph.D., Sarah Davis, Trevor Dundon, Sarah Dunn, Ph.D., Terry Maratos-Flier, M.D., Asma Haq, Carolyn Kloek, Hugh Lavery, Michael Schwartz, M.D., and Walter Stearns for their many invaluable contributions to the work that I have described. Supported by NIH DK56731 and DK57768 and grants from the American Diabetes Association.

\section{REFERENCES}

Ahima RS, Prabakaran D, Mantzoros CS 1996 Role of leptin in the neuroendocrine response to fasting. Nature 382:250-252

Araki E, Lipes MA, Patti ME 1994 Alternative pathway of insulin signalling in mice with targeted disruption of the IRS-1 gene. Nature 372:186-190 
Banks AS, Davis SM, Bates SH 2000 Activation of downstream signals by the long form of the leptin receptor. J Biol Chem 275:14563-14572

Bates SH, Steams WH, Schubert M 2003 STAT3 signaling is required for leptin regulation of energy balance but not reproduction. Nature 421:856-859

Baumann H, Morella KK, White DW 1996 The full-length leptin receptor has signaling capabilities of interleukin 6-type cytokine receptors. Proc Natl Acad Sci USA 93:8374-8378

Bjorbaek C, Uotani S, da Silva B 1997 Divergent signaling capacities of the long and short isoforms of the leptin receptor. J Biol Chem 272:32686-32695

Bjorbaek C, Elmquist JK, Frantz JD 1998 Identification of SOCS-3 as a potential mediator of central leptin resistance. Mol Cell 1:619-625

Bjorbaek C, Lavery HJ, Bates SH 2000 SOCS3 mediates feedback inhibition of the leptin receptor via Tyr985. J Biol Chem 275:40649-40657

Bjorbaek C, Buchholz RM, Davis SM 2001 Divergent roles of SHP-2 in ERK activation by leptin receptors. J Biol Chem 276:4747-4755

Bornstein SR, Uhlmann K, Haidan A 1997 Evidence for a novel peripheral action of leptin as a metabolic signal to the adrenal gland: leptin inhibits cortisol release directly. Diabetes 46:1235-1238

Bruning JC, Gautam D, Burks DJ 2000 Role of brain insulin receptor in control of body weight and reproduction [see comments]. Science 289:2122-2125

Burcelin R, Kamohara S, Li J 1999 Acute intravenous leptin infusion increases glucose turnover but not skeletal muscle glucose uptake in oblob mice. Diabetes 48:1264-1269

Chua SC Jr, Chung WK, Wu-Peng XS 1996 Phenotypes of mouse diabetes and rat fatty due to mutations in the OB (Leptin) receptor. Science 271:994-996

Chua SC Jr, Koutras IK, Han L 1997 Fine structure of the murine leptin receptor gene: splice site suppression is required to form two alternatively spliced transcripts. Genomics 45:264-270

Clement K, Vaisse C, Lahlou N 1998 A mutation in the human leptin receptor gene causes obesity and pituitary dysfunction. Nature 392:398-401

Considine RV, Sinha MK, Heiman ML 1996 Serum immunoreactive-leptin concentrations in normal-weight and obese humans. N Engl J Med 334:292-295

Cowley MA, Smart JL, Rubinstein M 2001 Leptin activates anorexigenic POMC neurons through a neural network in the arcuate nucleus. Nature 411:480-484

De Souza D, Fabri LJ, Nash A 2002 SH2 domains from suppressor of cytokine signaling-3 and protein tyrosine phosphatase SHP-2 have similar binding specificities. Biochemistry 41: 9229-9236

Elmquist JK, Bjorbaek C, Ahima RS 1998 Distributions of leptin receptor mRNA isoforms in the rat brain. J Comp Neurol 395:535-547

Elmquist JK, Elias CF, Saper CB 1999 From lesions to leptin: hypothalamic control of food intake and body weight. Neuron 22:221-232

Elmquist JK, Ahima RS, Maratos-Flier E 1997 Leptin activates neurons in ventrobasal hypothalamus and brainstem. Endocrinology 138:839-842

Elmquist JK, Maratos-Flier E, Saper CB 1998 Unraveling the central nervous system pathways underlying responses to leptin. Nature Neurosci 1:445-449

Erickson JC, Hollopeter G, Palmiter RD 1996 Attenuation of the obesity syndrome of ob/ob mice by the loss of neuropeptide Y. Science 274:1704-1707

Fan W, Boston BA, Kesterson RA 1997 Role of melanocortinergic neurons in feeding and the agouti obesity syndrome. Nature 385:165-168

Feng J, Witthuhn BA, Matsuda T 1997 Activation of Jak2 catalytic activity requires phosphorylation of $\mathrm{Y}^{1007}$ in the kinase activation loop. Mol Cell Biol 17:2497-2501

Flier JS 1995 The adipocyte: storage depot or node on the energy information superhighway. Cell $8: 15-18$ 
Frederich RC, Hamann A, Anderson S 1995 Leptin levels reflect body lipid content in mice: evidence for diet-induced resistance to leptin action. Nature Med 1:1311-1314

Friedman JM, Halaas JL 1998 Leptin and the regulation of body weight in mammals. Nature 395:763-770

Ge H, Huang L, Pourbahrami T 2002 Generation of soluble leptin receptor by ectodomain shedding of membrane-spanning receptors in vitro and in vivo. J Biol Chem 277:45898 45903

Grill HJ, Schwartz MW, Kaplan JM 2002 Evidence that the caudal brainstem is a target for the inhibitory effect of leptin on food intake. Endocrinology 143:239-246

Halaas JL, Gajiwala KS, Maffei M 1995 Weight-reducing effects of the plasma protein encoded by the obese gene. Science 269:543-546

Harvey J, McKay NG, Walker KS 2000 Essential role of phosphoinositide 3-kinase in leptininduced K(ATP) channel activation in the rat CRI-G1 insulinoma cell line. J Biol Chem 275:4660-4669

Ihle IN 1995 Cytokine receptor signalling. Nature 377:591-594

Ihle IN, Kerr IM 1995 Jaks and Stats in signaling by the cytokine receptor superfamily. Trends Genet 11:69-74

Inui A 1999 Feeding and body-weight regulation by hypothalamic neuropeptides - mediation of the actions of leptin. Trends Neurosci 22:62-67

Jureus A, Cunningham MJ, McClain ME 2000 Galanin-like peptide (GALP) is a target for regulation by leptin in the hypothalamus of the rat. Endocrinology 141:2703-2706

Kieffer TJ, Heller RS, Leech CA 1997 Leptin suppression of insulin secretion by the activation of ATP-sensitive $\mathrm{K}^{+}$channels in pancreatic beta-cells. Diabetes 46:1087-1093

Kloek C, Haq AK, Dunn SL 2002 Regulation of Jak kinases by intracellular leptin receptor sequences. J Biol Chem 277(44):41547-41555

Koch CA, Anderson DJ, Moran MF 1991 SH2 and SH3 domains: elements that control interactions of cytoplasmic signaling proteins. Science 252:668-674

Kulkarni RN, Wang ZL, Wang RM 1997 Leptin rapidly suppresses insulin release from insulinoma cells, rat and human islets and, in vivo, in mice. J Clin Invest 100:2729-2736

Licinio J, Mantzoros C, Negrao AB 1997 Human leptin levels are pulsatile and inversely related to pituitary-adrenal function. Nature Med 3:575-579

Liu L, Karkanias GB, Morales JC 1988 Intracerebroventricular leptin regulates hepatic but not peripheral glucose fluxes. J Biol Chem 273:31160-31167

Lord GM, Matarese G, Howard JK 1998 Leptin modulates the T-cell immune response and reverses starvation-induced immunosuppression. Nature 394:897-901

Lowenstein EJ, Daly RJ, Batzer AG 1992 The SH2 and SH3 domain-containing proteins GRB2 links receptor tyrosine kinases to ras signaling. Cell 70:431-442

MacDougald OA, Hwang CS, Fan H 1995 Regulated expression of the obese gene product (leptin) in white adipose tissue and 3T3-L1 adipocytes. Proc Natl Acad Sci USA 92:9034-9037

Maffei M, Halaas J, Ravussin E 1995 Leptin levels in human and rodent: measurement of plasma leptin and $o b$ RNA in obese and weight-reduced subjects. Nature Med 1:1155-1161

Montague CT, Farooqi IS, Whitehead JP 1997 Congenital leptin deficiency is associated with severe early onset obesity in humans. Nature 387:903-908

Myers MG Jr, Wang LM, Sun XJ 1994 The role of IRS-1/GRB2 complexes in insulin signaling. Mol Cell Biol 14:3577-3587

Myers MG Jr, White MF 2002 The molecular basis of insulin action. In: DeGroot LJ, Jameson JL, eds. Endocrinology, edit 4. Philadelphia: Saunders; 712-727

Niswender KD, Morrison CD, Clegg DJ 2003 Insulin activation of phosphatidylinositol 3-kinase in the hypothalamic arcuate nucleus: a key mediator of insulin-induced anorexia. Diabetes $52: 227-231$ 
Niswender KD, Morton GJ, Stearns WH 2001 Intracellular signaling: key enzyme in leptininduced anorexia. Nature 413:794-795

Rahmouni K, Haynes WG, Morgan DA 2003 Intracellular mechanisms involved in leptin regulation of sympathetic outflow. Hypertension 41:763-767

Rentsch J, Chiesi M 1996 Regulation of ob gene mRNA levels in cultured adipocytes. FEBS Lett 379:55-59

Roth J 1998 Diabetes and obesity. Diab Metab Rev 13:1-2

Schwartz MW, Woods SC, Porte D Jr 2000 Central nervous system control of food intake. Nature 404:661-671

Seeley RJ, Yagaloff KA, Fisher SL 1997 Melanocortin receptors in leptin effects. Nature 390:349

Slieker LJ, Sloop KW, Surface PL 1996 Regulation of expression of $o b$ mRNA and protein by glucocorticoids and cAMP. J Biol Chem 271:5301-5304

Songyang Z, Shoelson SE, Chaudhuri M 1993 SH2 domains recognize specific phosphopeptide sequences. Cell 72:767-778

Spanswick D, Smith MA, Groppi VE 1997 Leptin inhibits hypothalamic neurons by activation of ATP-sensitive potassium channels. Nature 390:521-525

Taga T, Kishimoto T 1997 gp 130 and the interleukin-6 family of cytokines. Ann Rev Immunol 15:797-819

Tartaglia LA 1997 The leptin receptor. J Biol Chem 272:6093-6096

Tartaglia LA, Dembski M, Weng X 1995 Identification and expression cloning of a leptin receptor, OB-R. Cell 83:1263-1271

White DW, Kuropatwinski KK, Devos R 1997 Leptin receptor (OB-R) signaling. J Biol Chem 272:4065-4071

Withers DJ, Gutierrez JS, Towery H 1998 Disruption of IRS-2 causes type 2 diabetes in mice. Nature 391:900-904 